\title{
Données hydrogéologiques préliminaires au projet d'une exploitation de la nappe albienne fondée sur l'alimentation artificielle
} (Région parisienne)

\author{
par J. Bize \\ Bureau d'Etudes BURGEAP
}

\section{Les bases du projet}

Sous l'impulsion d'Arago, qui prévoyait le passage des sables de l'Albien sous la craie parisienne, et avec les crédits du Conseil Municipal de Paris, l'entrepreneur Mulot commençait à Grenelle, le 24 décembre 1833, un forage dont la profondeur prévue était de $400 \mathrm{~m}$. Ce n'est que sept ans plus tard que les "sables verts " furent atteints, à la profondeur de $548 \mathrm{~m}$. L'eau jaillit à $33 \mathrm{~m}$ de hauteur au débit de $160 \mathrm{~m}^{3} / \mathrm{h}$. Le niveau piézométrique calculé s'élevait à la cote 128 NGF. En 1861, un deuxième forage, celui de Passy (Square Lamartine) atteignait la nappe à $528 \mathrm{~m}$ de profondeur, et débitait le premier jour $20000 \mathrm{~m}^{3}$ d'eau.

Actuellement, on compte 42 puits forés à l'Albien dans la région parisienne; 33 restent en activité et 5 seulement sont encore artésiens. Car, sur environ un siècle, le niveau piézométrique de la nappe baissa à Paris d'une centaine de mètres, sous l'effet d'une exploitation relativement intensive, qui culmina à un peu moins de $1,2 \mathrm{~m}^{3} / \mathrm{s}$ entre 1934 et 1936

En 1935, on jugea nécessaire de prendre des mesures restrictives et un décret-loi stoppa la prolifération des forages, dont la construction fut désormais soumise au régime de l'autorisation préalable. Les effets de ce décret, joints à ceux d'un "vieillissement » progressif des forages existants, ont ramené actuollement le débit d'exploitation à la valeur moyenne de $0,7-0,8 \mathrm{~m}^{3} / \mathrm{s}$; parallèlement, si on constate que l'abaissement du niveau de la nappe est encore notable dans les zones de forte exploitation, au centre et au nord de Paris, il est négligeable dans la partie est. Le niveau piézométrique se situe actuellement dans la région parisienne entre 15 et $30 \mathrm{NGF}$

La politique amorcée en 1935 atteint donc ses objectifs. Mais dans le même temps, ou plutôt durant la dernière décennie, l'évolution des données du problème de l'eau et des mentalités avait pour résultat de valoriser les qualités de la nappe albienne: présence en continu sous le tissu urbain très serré de la région parisienne d'une nappe,

- contenant des eaux d'excellentes caractéristiques,

- bien protégée des pollutions,

- fournissant des débits ponotuels intéressants (de l'ordre de $200 \mathrm{~m}^{3} / \mathrm{h}$ ),

- très "nerveuse ", c'est-à-dire capable de transmettre rapidement des pressions,

- enfin, ne nécessitant pas des frais d'exhaure élevés (compte tenu de la faible profondeur du niveau piézométrique).

Au début des années 60, après une longue période de sommeil, on note, de manière significative, un regain d'activité autour de la nappe albienne: notamment étude du Service de la Conservation des Gisements $\left({ }^{a}\right)$, thèse de M. Lauverjat $\left(^{b}\right)$.

En même temps, les perspectives offertes par l'alimentation artificielle des nappes, tout à fait nouvelles à l'époque en France, rendent plausible un recours accru aux ressources de la nappe sans remettre en cause le principe contenu dans le décret de 1935, qui vise la conservation des niveaux dans la zone de forte exploitation que constitue la région parisienne $\left({ }^{c}\right)$.

En 1966, la Compagnie Générale des Eaux, en liaison avec l'Agence Financière de Bassin Seine-Normandie, for-

(a) «Contribution à l'étude hydrogéologique de la nappe a'bienne dans le bassin de Paris $»$ (janvier 1965). Cette étude a été présentée dans les Annales des Mines de mai 1966 par C. Sarocchi et H. LÉVY-LAMBERT.

(b) «Contribution à l'étude géologique et hydrogéologique de l'Albien dans le centre du Bassin de Paris». Thèse publiée par le B.R.G.M. en mars 1967.

(c) Dans son étude, le Service de la Conservation des Gisements envisage l'hypothèse d'une recharge dans la région d'Epernay, à plus de $100 \mathrm{~km}$ de Paris. 
mule l'idée que des doublets d'injection et de pompage pourraient être mis en cuvre dans la région parisienne. Ces doublets auraient pour effet d'y moduler (en " creux" et en "bosses») la surface piézométrique de la nappe, sans modifier les conditions de son abaissement.

Implantés près des usines de traitement ou des principales antères de réseaux, les forages d'injection seraient alimentés, en continu ou seulement en période de faible demande, en eaux de surface convenablement traitées.

Les forages de pompage seraient utilisés pour l'alimentation temporaire des zones urbaines nouvellement créées, puis pour fournir une ressource d'appoint ou de sécurité.

Dans ce dispositif, la nappe jouerait donc le rôle de vecteur et de modulateur de la ressource en eau potable $\left(^{d}\right)$.

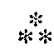

Dès 1966, la Compagnie Générale des Eaux entreprenait, pour le compte du Syndicat des Communes de la Banlieue de Paris pour les Eaux, des études sur la faisabilité du projet. L'hydrogéologie était confiée à BURGEAP, la physico-chimie des eaux à la SETUDE.

Dans l'étude hydrogéologique $\left({ }^{e}\right)$, on pouvait distinguer deux parties:

- la prévision des effets à distance de pompages et d'injections, afin de "tester" différentes hypothèses où entrent en compte, d'une part la distance entre les forages d'un doublet, d'autre part le programme de pompage et d'injection,

- la réalisation d'essais d'injection avec, pour principal objectif, l'étude du colmatage.

Les recherches de la première partie ont été fondées sur l'exploitation des données existantes et sur la possibilité qui s'est présentée en cours d'étude de réaliser un pompage d'essai en profitant de la remise en service du forage de Villemomble (Ville de Paris) avec observation des rabattements sur les forages voisins, notamment celui de Noisy-le-Grand, appartenant à la C.G.E., sur lequel il était prévu de réaliser des essais d'injection. Bien entendu, afin de rendre significatives les variations de pression enregistrées, il a été nécessaire - considérant qu'en période d'essai tout forage, sauf le forage d'essai, est à la fois le siège d'un pompage parasite et un piézomètre possible - de faire le tour de tous les forages de la région parisienne afin de connaître leur régime normal d'exploitation et de pression, les possibilités d'y effectuer des mesures, etc.

Connaissant les paramètres hydrauliques de la nappe, il a été possible de tester différentes hypothèses d'injection-pompage. Il reste beaucoup à faire dans ce domaine, tant qu'un modèle de simulation des écoulements de la nappe, actuellement ébauché, ne sera pas réalisé.

Les essais d'injection - suivis de repompages - ont été effectués sur le forage de Noisy, avec des eaux de Marne traitées à l'usine de Neuilly-sur-Marne: l'un de quinze jours en juillet 1970, l'autre de six mois, de janvier à juil-

(d) Les aspects économiques du projet seront présentés dans un prochain numéro de Technique et Sciences Municipales: L'esquichage de la nappe albienne en Région parisienne par P. L. Girardot et M. Kester.

(e) Qui fait l'objet de cet exposé à l'exclusion des questions concernant la compatibilité entre eaux d'injection et eaux de gisement, prétraitement, maintien de la qualité, qui sont du ressort de ia SETUDE. let 1973. Le repompage, qui doit s'étaler sur dix mois, est en cours.

Nous présenterons rapidement les principaux résultats de l'étude; nombre d'entre eux ne sont pas encore interprétés et doivent être considérés comme provisoires. Mais auparavant nous ferons un retour sur les caractéristiques essentielles du gisement.

\section{Généralités sur la nappe de l'Albien}

\author{
Extension. Profondeur.
}

L'Albien du Bassin de Paris s'étend sur $75000 \mathrm{~km}^{2}$ environ (fig. 1). A Meaux, au centre de la cuvette du bassin parisien, sa profondeur atteint le maximum de $750 \mathrm{~m}$.

\section{La couche aquifère (fig. 2)}

L'Albien est composé d'une alternance de grès fins, peu ou pas consolidés, et de bancs argileux ou argilo-sableux.

Complétant l'ouvrage de base de Lemoine, Humery et Soyer, publié en 1936, l'étude du Service de la Conservation des Gisements, basée sur les renseignements fournis par les nombreux forages pétroliers, a conduit à définir à partir des $《 \log »$ de carottages électriques, trois récurrences d'argile et de grès, à peu près constantes dans l'ensemble du bassin parisien, et désignées sous le nom de "membres » argilo-gréseux supérieur, moyen et inféricur (AGS, AGM, AGI).

Dans chacun de ces membres, il a été possible de délimiter des couches de "grès utiles», constituant le réservoir aquifère proprement dit.

Dans la région parisienne, où l'épaisseur de la formation est d'une centaine de mètres, on peut admettre que l'essentiel de la transmissivité de l'Albien (produit de la perméabilité par l'épaisseur de la couche aquifère) se répartit sur $30 \mathrm{~m}$ d'épaisseur seulement (fig. 3), dont plus des deux tiers environ dans un ensemble non-subdivisé, constitué par l'AGS et 1'AGM.

\section{Les épontes ef les nappes encaissantes (fig. 4)}

\section{- Au ToIT :}

La nappe albienne est surmontée par une quarantaine de mètres d'argiles et de marnes (argiles de Gault et marnes de Brienne, d'après Lauverjat) qui la séparent de la nappe de la Craie dont la base, plus ou moins marneuse, appartient au Cénomanien. A l'origine, avant 1840, la nappe albienne était en charge de plus de $40 \mathrm{~m}$ par rappont à la nappe de la Craie.

- AU MUR:

Les formations argilo-sableuses de l'Albien reposent sur un niveau argileux homogène et constant d'âge aptien, bien caractérisé par de basses résistivités.

Cet Aptien sépare la nappe albienne des niveaux aquifères sableux et sablo-argileux du Barrémien supérieur et du Néocomien. 


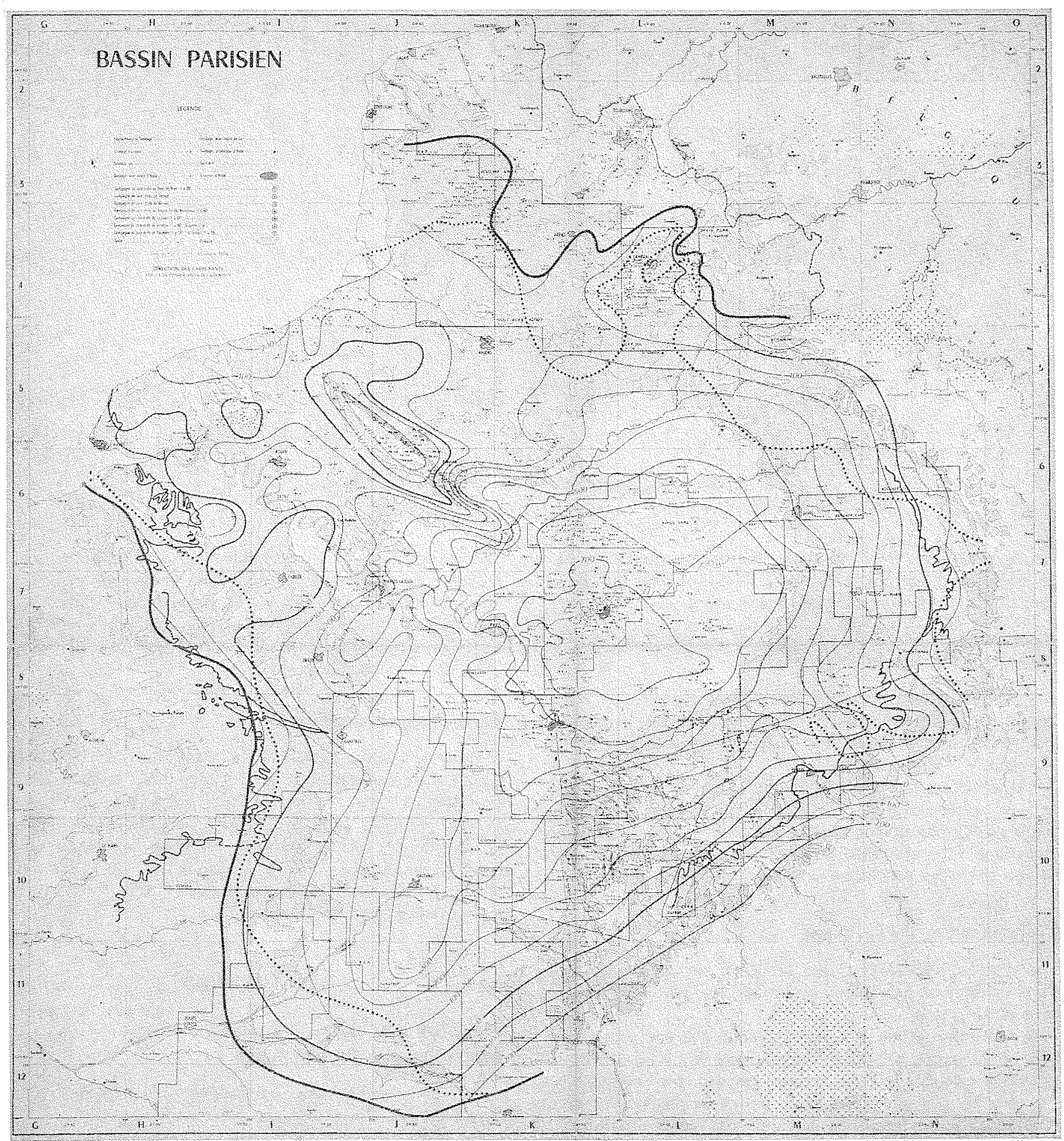

1/Isobathes du toit des grès Albien (d'après Service de Conservation des Gisements). La limite des grès " utiles" est illustrée par des pointillés. 


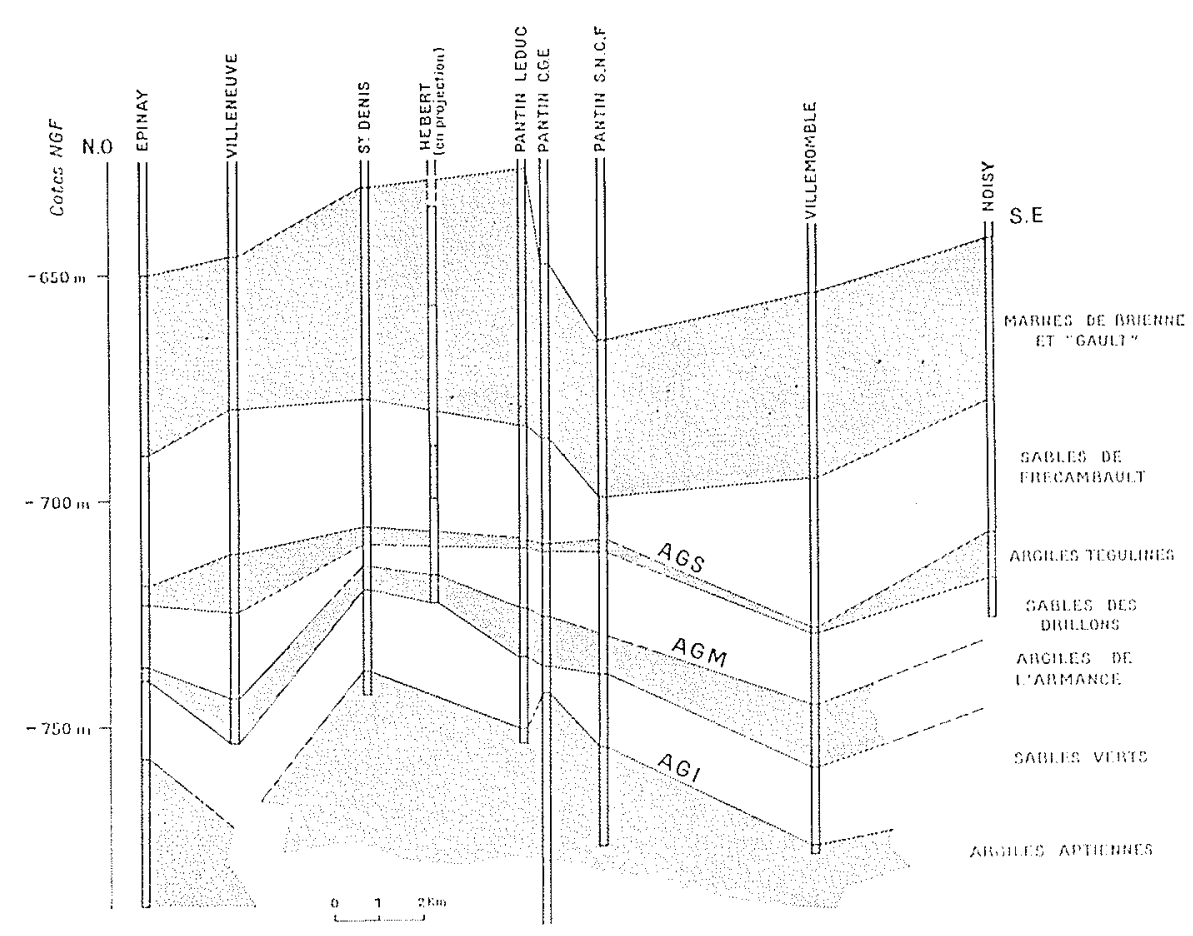

2/ Coupe géologique NO-SE de l'Albien à Paris.

L'épaisseur des grès utiles du Barrémien supérieur est de $10 \mathrm{~m}$ pour une épaisseur totale de $50 \mathrm{~m}$, celle du Néocomien de $35 \mathrm{~m}$ pour une épaisseur totale de $90 \mathrm{~m}$.

\section{Données structurales}

Les assises sédimentaires du bassin parisien se sont déposées sur un substratum hercynien, dont les fractures ont rejoué tout au long des temps géologiques et principalement à l'ère tertiaire.

Ces mouvements tectoniques ont engendré dans la couverture sédimentaire une série d'ondulations, parfois faillées, de direction générale N.O.-S.E. Dans la région parisienne, le toit des couches albiennes s'enfonce de - $500 \mathrm{NGF}$ au S.O. à $-700 \mathrm{NGF}$ au N.E.

\section{L'alimentation de la nappe}

L'alimentation de la nappe constitue un problème qui, s'il a été éclairci ces dernières années, n'a pas encore reçu de réponse nette.

D'après une étude isotopique récente $(f)$, une alimentation de la nappe existerait essentiellement sur sa bordure aux affleurements ou au travers du toit peu perméable du réservoir, lorsqu'il n'effleure pas. Ces résultats, purement qualitatifs, notons-le, ne sont pas en contradiction avec des calculs, effectués avec des données assez sûres, qui nous ont amené à conclure que dans la région de forte exploitation de Paris et de sa banlieule, les échanges entre l'Albien et la Craie sus-jacente, s'ils peuvent exister, ne pourraient être que tout à fait négligeables.

(f) Y. Vurllaume : Utilisation des méthodes isotopiques et hydrochimiques en hydrogéologie. Application à l'étude de la nappe de l'A'bien dans le bassin de Paris. B.R.G.M., août 1971.
$\mathrm{Au}$ contraire, il est probable que les aquifères sousjacents, Barremien et Néocomien, réagissent solidairement avec l'Albien lors de pompages ou d'injections. L'étude en cours devrait permettre d'éclaircir ce point, dont l'importance est évidente pour la prévision des variations de pression à distance.

\section{Paramètres hydrauliques}

Dans la région parisienne la transmissivité et le coefficient d'emmagasinement de l'Albien sont respectivement de $1 \cdot 10^{-3}$ à $5 \cdot 10^{-3} \mathrm{~m}^{2} / \mathrm{s}$ et $1 \cdot 10^{-4}$ à $2,5 \cdot 10^{-4}$.

Une première approche, fondée sur des extrapolations de courbe de rabattement (fig. 5), donc à prendre avec réserve, montrerait que la transmissivité de l'ensemble Albien, Barrémien, Néocomien pourrait être de $4.10^{-3} \mathrm{~m}^{2} / \mathrm{s}$, le coefficient d'emmagasinement de $2,8.10^{-4}$.

\section{Les résultats}

\section{Prévision des effets à distance d'injections et de pompages}

La connaissance des paramètres hydrauliques de la nappe permet d'effectuer ces prévisions.

Le phénomène de l'injection est, pour la nappe, l'inverse de celui du pompage. $\mathrm{Si}$ besoin était, une vérification pouvait en être fournie par les remontées observées sur le forage de Villemomble, distant de $4,1 \mathrm{~km}$ de celui de Noisy-le-Grand sur lequel un débit de $115 \mathrm{~m}^{3} / \mathrm{h}$ a été injecté. Ces remontées confirment, en effet, les calculs. On note, par exemple, qu'elles sont de $2 \mathrm{~m}$ au bout de six mois (fig. 6).

Deux cas d'injection-pompage ont été étudiés. 


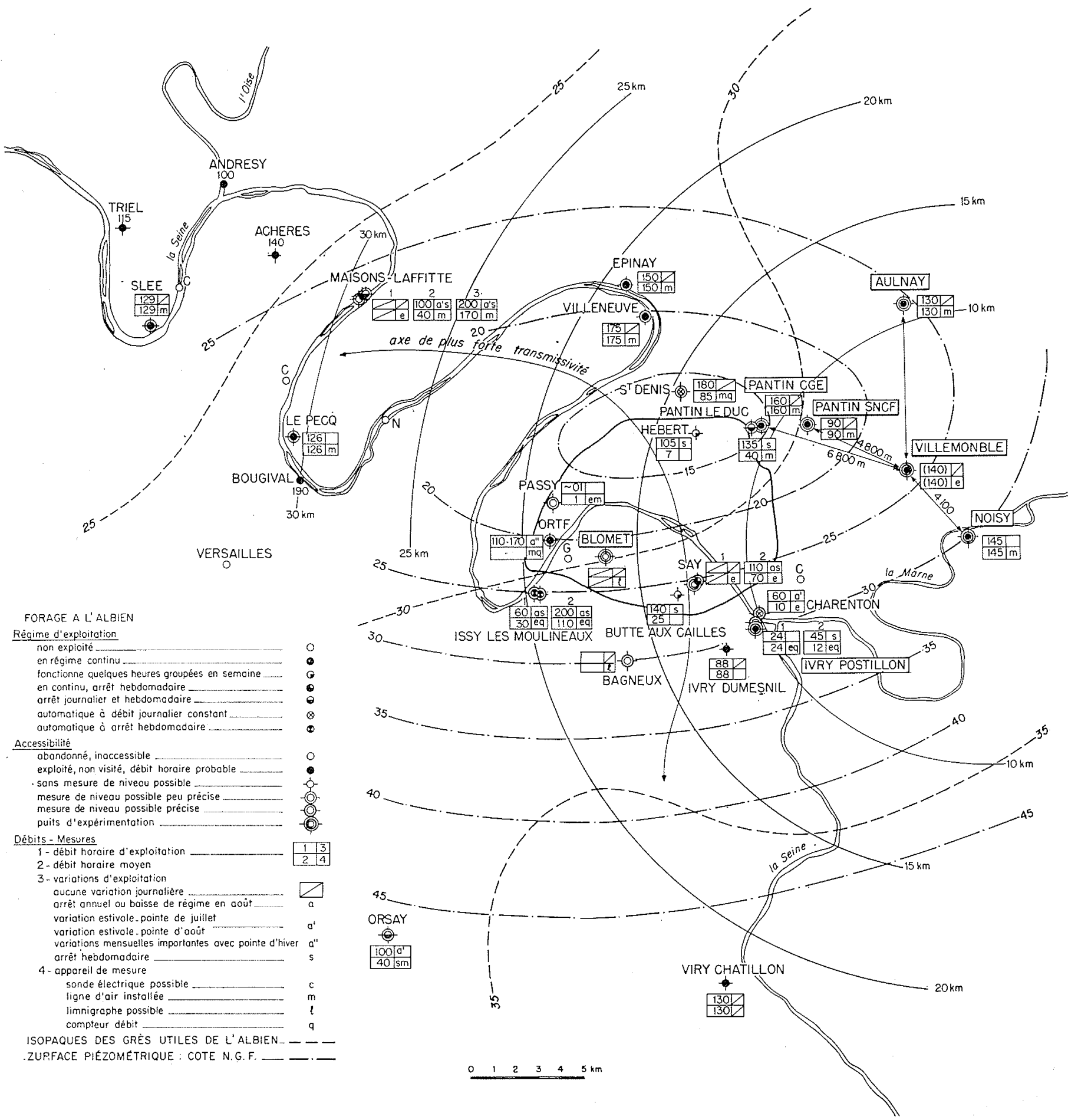

3/ Etude de l'alimentation artificielle de la nappe albienne.

Exploitation de la nappe albienne dans la Région parisienne. Dispositif d'expérimentation. 


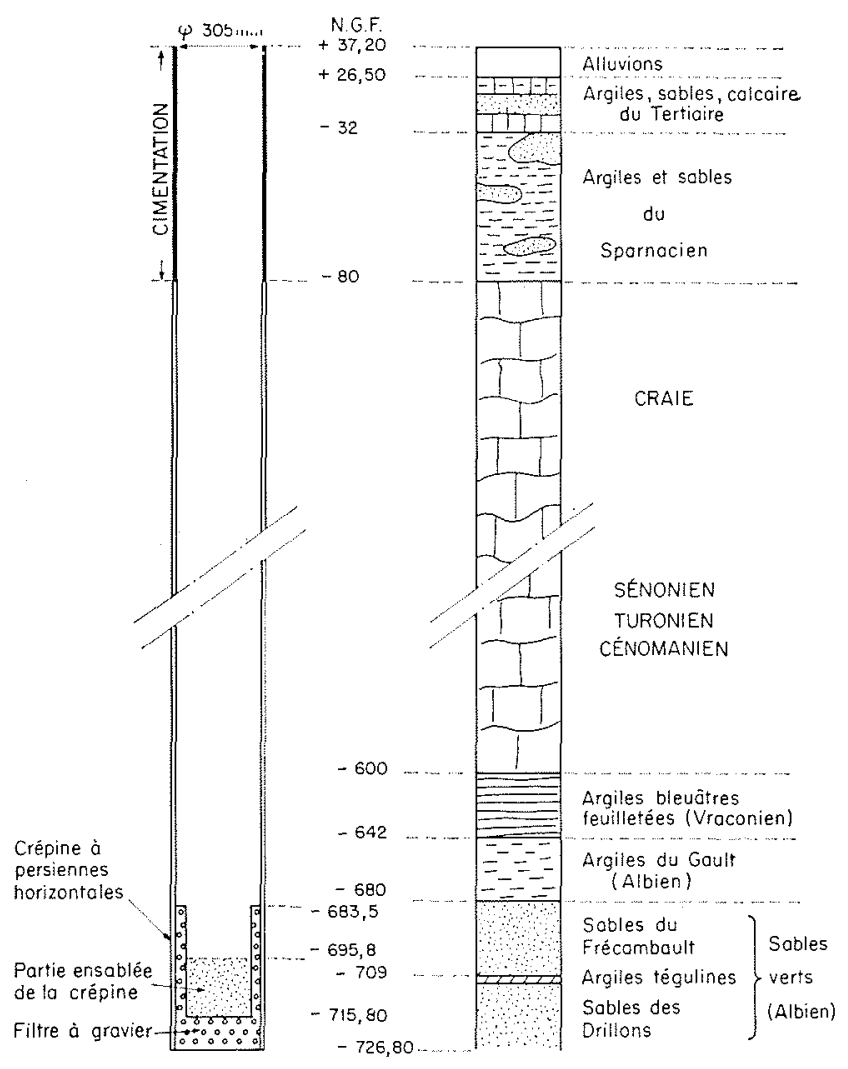

4/ Forage de Noisy-le-Grand. Coupe technique ef géologique.

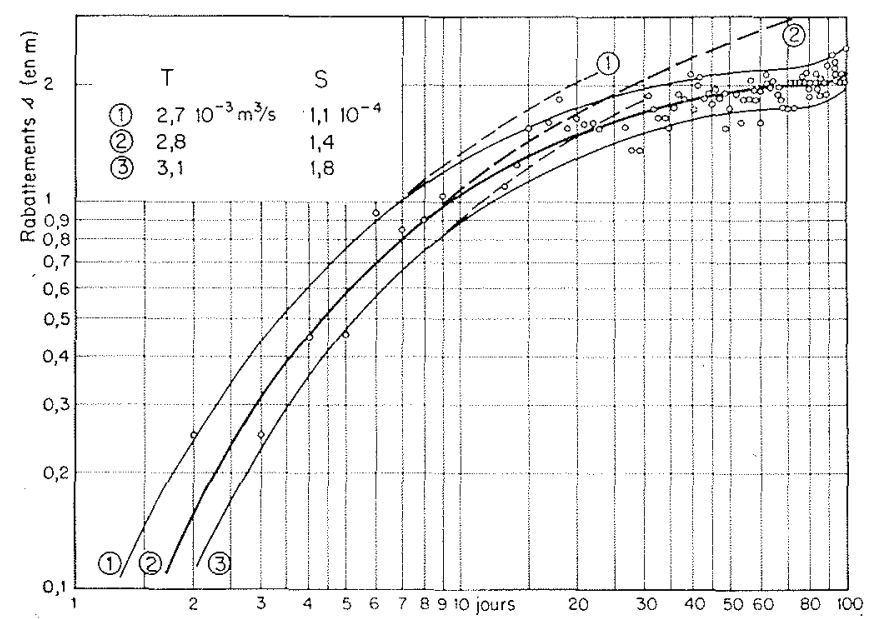

5/ Rabattement ati forage de Noisy-le-Grand provoqué par un pompage de $140 \mathrm{~m}^{3 / h}$ sur le forage de Villemonble. Drainance probable à partir du $10^{\circ}$ jour.

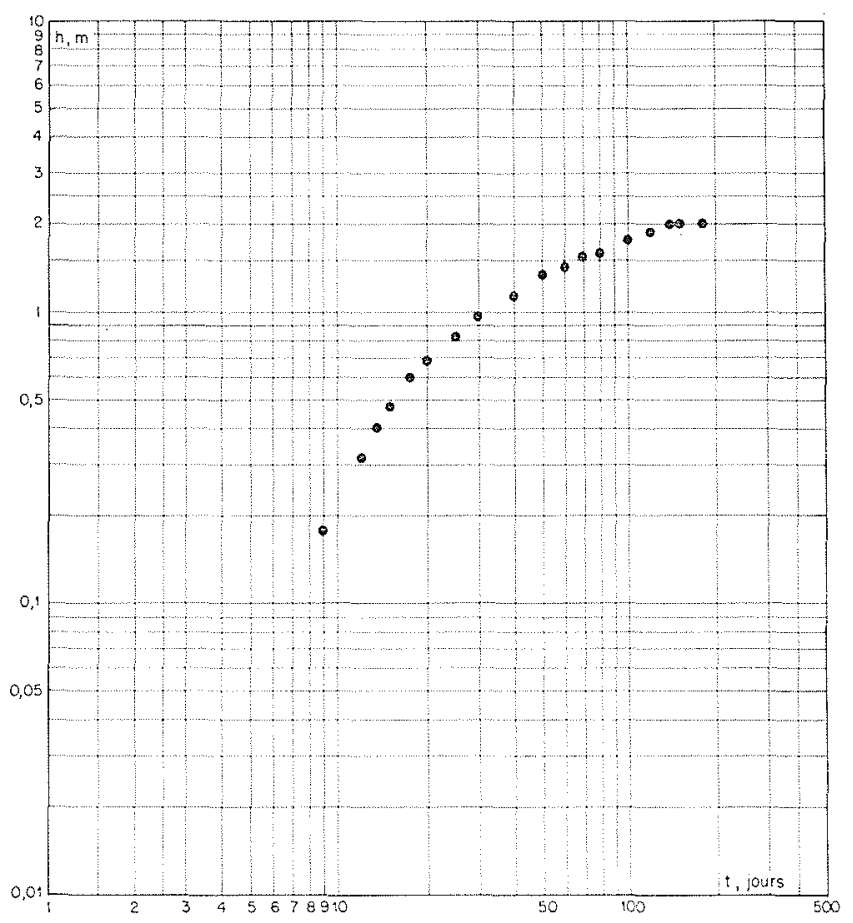

6/ Remontée de pression au torage de Villemomble au cours d'une injection de 6 mois au débit de $115 \mathrm{~m}^{3} / \mathrm{h}$ sur le forage de Noisy-le-Grand, distant de $4,1 \mathrm{~km}$.

- Premier cas (fig. 7):

Ce cas est constitué d'un doublet de forages distants de $10 \mathrm{~km}$. La recharge et le prélèvement sont effectués simultanément au débit de $200 \mathrm{~m}^{3} / \mathrm{h}$. On constate que ce système perturbe très peu une surface piézométrique supposée initialement plane. En effet, les variations de cette surface sont, après 1,10 ou 100 ans d'exploitation, inférieures à $3 \mathrm{~m}$ dès que l'on s'écarte de $5 \mathrm{~km}$ des forages.

L'utilisation d'un tel doublet semble particulièrement indiquée lorsqu'on désire compenser, par des injections intermédiaires, les effets de nouveaux pompages effectués à la périphérie d'une zone centrale de forts prélèvements. Dans ce cas, la surface piézométrique initiale est pentue et le remodelage qu'elle subit a pour effet de raboter les collines piézométriques qui caractérisent les régions jusqu'ici peu sollicitées, en comblant le flanc de la cuvette piézométrique que représente la zone de fortes sollicitations, ce qui va dans le sens d'un certain nivellement.

La distance de $10 \mathrm{~km}$ entre les forages d'un doublet apparaît done comme un seuil à ne pas dépasser si l'on ne veut pas perturber outre mesure le niveau piézométrique de la nappe. Or, on a montré par ailleurs (cf. étude citée de P. L. Girardot et Kester) que cette distance était largement compatible avec l'économie des projets de doublets, la distance minimale de rentabilité étant de $7,5 \mathrm{~km}$ ou même de $3,5 \mathrm{~km}$ seulement, selon les cas. 
ÉVOLUTION DE LA SURFACE PIÉZOMETRIQUE INITIALEMENT PLANE APRES IO ANS

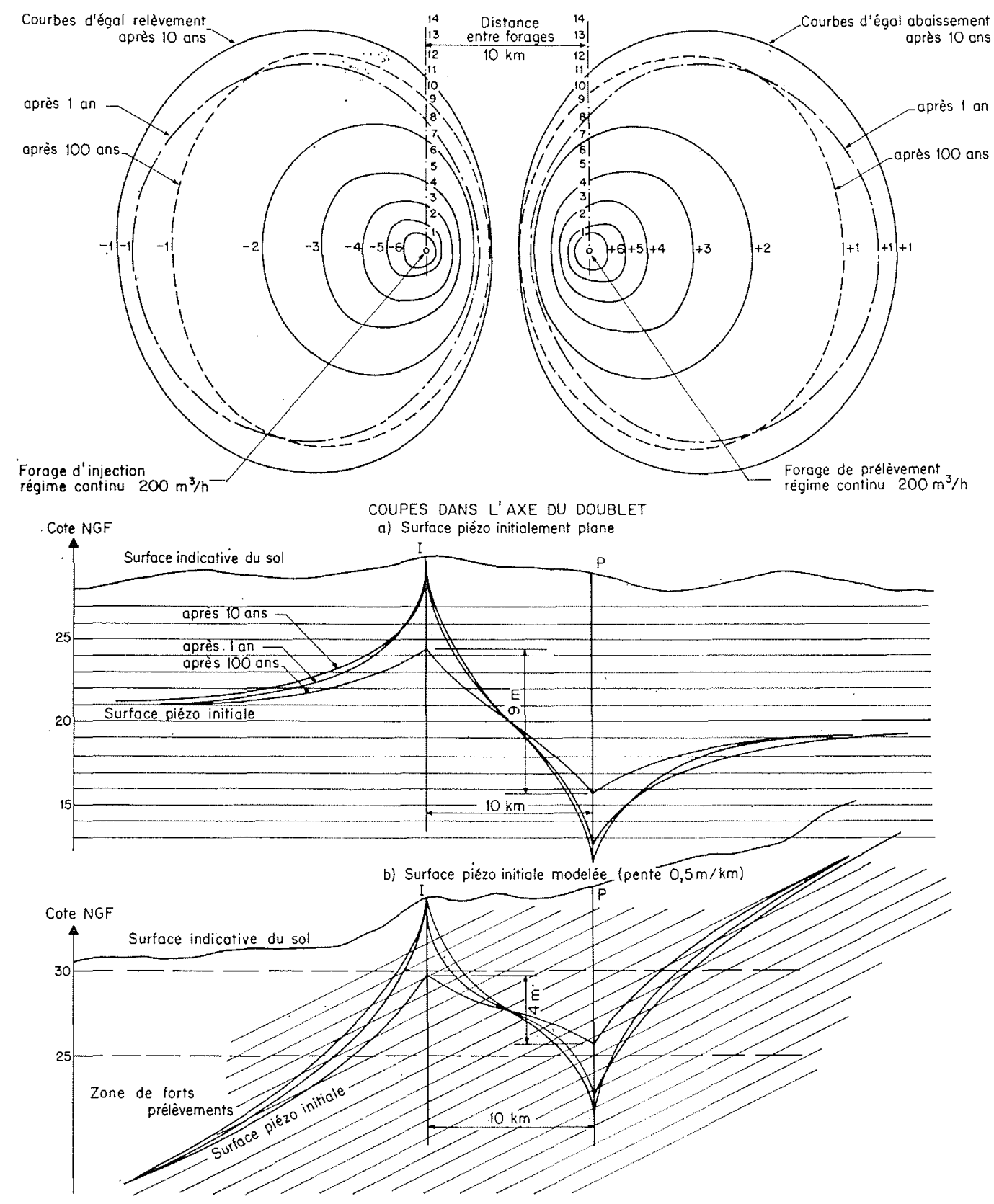

7/ Doublet de forages à l'Albien

- Deuxième cas (fig. 8) :

Le cas consiste en quatre forages de prélèvement, exploités en continu à $150 \mathrm{~m}^{3} / \mathrm{h}$, et placés à $5 \mathrm{~km}$ d'un forage central dans lequel on injecte $600 \mathrm{~m}^{3} / \mathrm{h}$. Cette étude montre que dans un tel système la surface piézométrique est très peu perturbée et que, notamment, autour de la maille constituée par les quatre forages de prélèvement la variation du niveau piézométrique ne dépasse pas $0,50 \mathrm{~m}$.

\section{Essai d'injection de six mois}

L'essai d'injection s'est déroulé sur le forage de la C.G.E. à Noisy-le-Grand, à proximité de l'usine de traitement de
Neuilly-sur-Marne. Effectué au débit continu de $115 \mathrm{~m}^{3} / \mathrm{h}$, il a duré du 22 janvier au 24 juillet 1973, soit durant six mois. Le volume d'eau injectée s'est élevé à $490000 \mathrm{~m}^{3}$ environ.

\section{Situation et données locales}

Exécuté en 1933 le forage de Noisy se situe à l'extrémité est de la zone à forte exploitation de la région parisienne. Placé à peu près en même situation, le forage le plus proche est celui de Villemonble, à $4100 \mathrm{~m}$ au N.O. (fig. 3).

Le forage de Noisy, qui ne capte que la partie supérieure de l'Albien, est profond de $764 \mathrm{~m}$. Les principales cotes NGF sont les suivantes (fig. 4). 
- Tête de puits : 41,05 ,

- Sol : 37,20,

- Crépine : - 683,5 à - 715,80.

La hauteur crépinée est donc de $32 \mathrm{~m}$, mais, compte tenu de l'ensablement du forage, qui forme un dépôt de $20 \mathrm{~m}$, il ne reste que $12 \mathrm{~m}$ de crépine libre (notons toutefois que le sable de dépôt est probablement un sable propre, donc plus fottement perméable que le terrain en place).

Avant essai, après plusieurs mois sans exploitation, le niveau piézométrique se situait à la cote 29,65 , soit $11,40 \mathrm{~m}$ sous la tête du forage.

Les paramètres hydrauliques déduits de l'essai de pompage réalisé en 1970 sur Villemomble sont, à Noisy, de $2,8.10^{-3} \mathrm{~m}^{2} / \mathrm{s}$ pour la transmissivité, de $1,4.10^{-4}$ pour le coefficient d'emmagasinement.

\section{Données de l'essai (fig. 9)}

\section{- Prétraitement particulier :}

Durant toute la durée de l'essai, l'eau potable fournie par l'usine de Neuilly subissait une correction de $\mathrm{pH}$ afin d'être à l'équilibre (ni entartrante, ni agressive) aux conditions de température et de pression régnant au niveau de la orépine, soit $32^{\circ} \mathrm{C}$ et environ 70 bars.

Par ailleurs, durant les trois premiers mois l'eau injectée a été surchlorée (puis déchlorée) afin d'être stérilisée (cf. étude de la SETUDE et contrôle effectué par les services de la Ville de Paris).

\section{- Equipement dU forage:}

A l'intérieur du tubage $(\varnothing 305 \mathrm{~mm})$ étaient disposés à demeure :

- une pompe immergée de reprise,

- le flotteur d'un limnigraphe R.16 OTT,

- le tube d'un «bulle à bulle »,

- enfin, deux tubes d'injection $\varnothing 60$, plongeant sous le niveau de l'eau et reliés par un $\varnothing 150$ à la pompe d'injection en surface (photo A). L'existence et le dimensionnement de ces tubes étaient justifiés par la nécessité de maintenir en permanence, en tout point du circuit d'injection, des pressions positives afin d'éviter l'entraînement d'air et de permettre la prise d'échantillons. Au débit d'injection prévu, soit $115 \mathrm{~m}^{3} / \mathrm{h}$, les tubes étaient en service; si nécessaire, en cas de réduction de débit, une vanne permettait de n'utiliser que l'un d'entre eux.

Notons, par ailleurs, que l'appareil enregistreur du limnigraphe était placé soit dans la coupole du forage, soit au-dessus à $6 \mathrm{~m}$ du sol, lorsque le niveau dynamique s'élevait au-dessus de la tête de forage, que l'on pouvait fermer (photo B).

\section{- Appareillage de mesures :}

Les mesures suivantes étaient effectuées en continu: débit et volume d'injection, niveau dynamique dans le forage, pression dans les conduites, $\mathrm{pH}$, résistivité et température de l'eau.

Le déroulement de l'essai pouvait être suivi sur un tableau de commande et de visualisation placé dans un

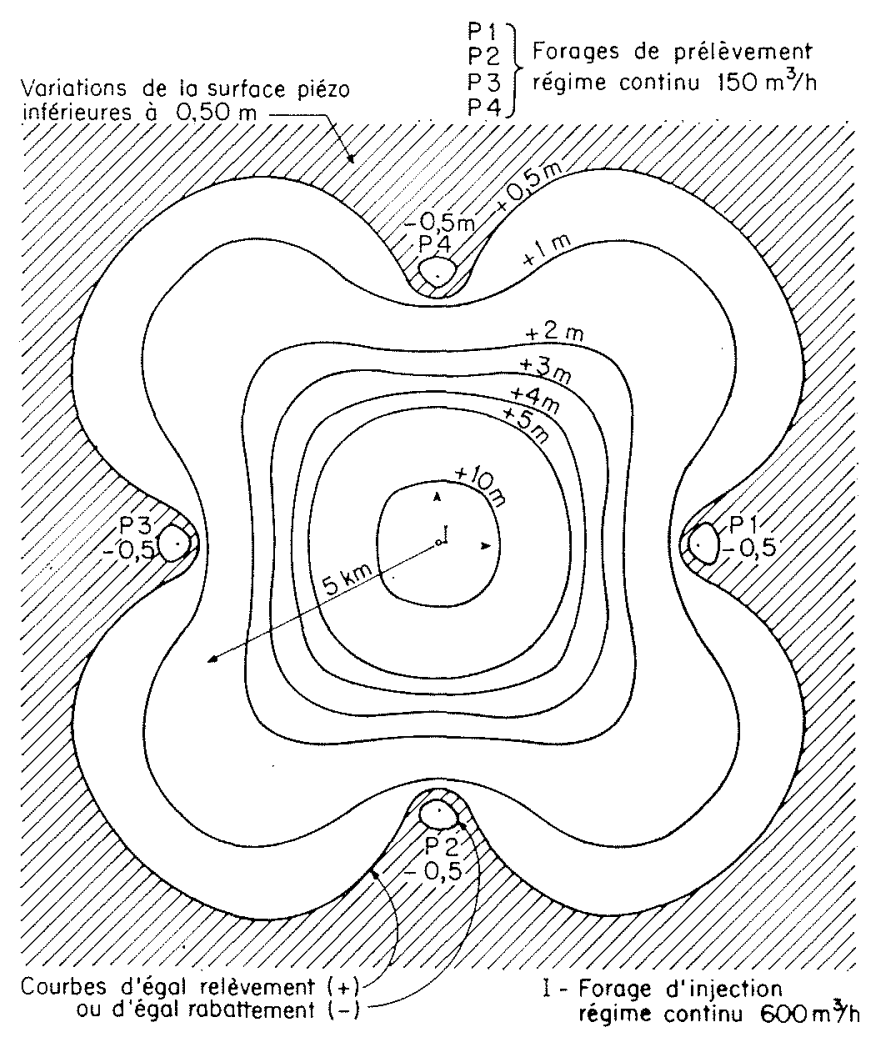

8/ Représentation d'un système équilibré injection-prélèvements. Evolution de la surface piézométrique, initialement plane, après 10 ans.

abri à proximité du forage (photo B) ("). Enfin, certains appareils étaient reliés au PC de l'usine de Neuilly, où fonctionnait un système d'alarmes (débit, niveaux, pH).

\section{- Paramètres et parasites de L'essai:}

L'objectif premier de l'essai était, rappelons-le, de mettre en évidence l'existence éventuelle d'un colmatage. Théoriquement, la marche à suivre consistait simplement à comparer la courbe de relèvement expérimentale à celle qui découle des pertes de charge dans l'aquifère, c'est-à-dire déduite des paramètres hydrauliques - connus - de la nappe.

En réalité, on devait tenir compte, en outre:

- des pertes de charge particulières provenant du tubage et de la crépine, et du fait que le forage est «imparfait ", et du gain de charge découlant du fait que, sur un rayon très probablement important (plusieurs dizaines de mètres?) autour de la crépine, les sables de l'aquifère sont délavés et possèdent localement une transmissivité plus élevée que celle de l'aquifère;

- de la baisse séculaire de la nappe (mais négligeable à Noisy);

- de la drainance (aquifères du Barremien et du Néocomien);

- des variations de pression provoquées par des variations du débit d'exploitation sur les forages de la région

(g) L'abri contenait en outre la pompe d'injection, l'unité d'acidification et le filtre de déchloration. 


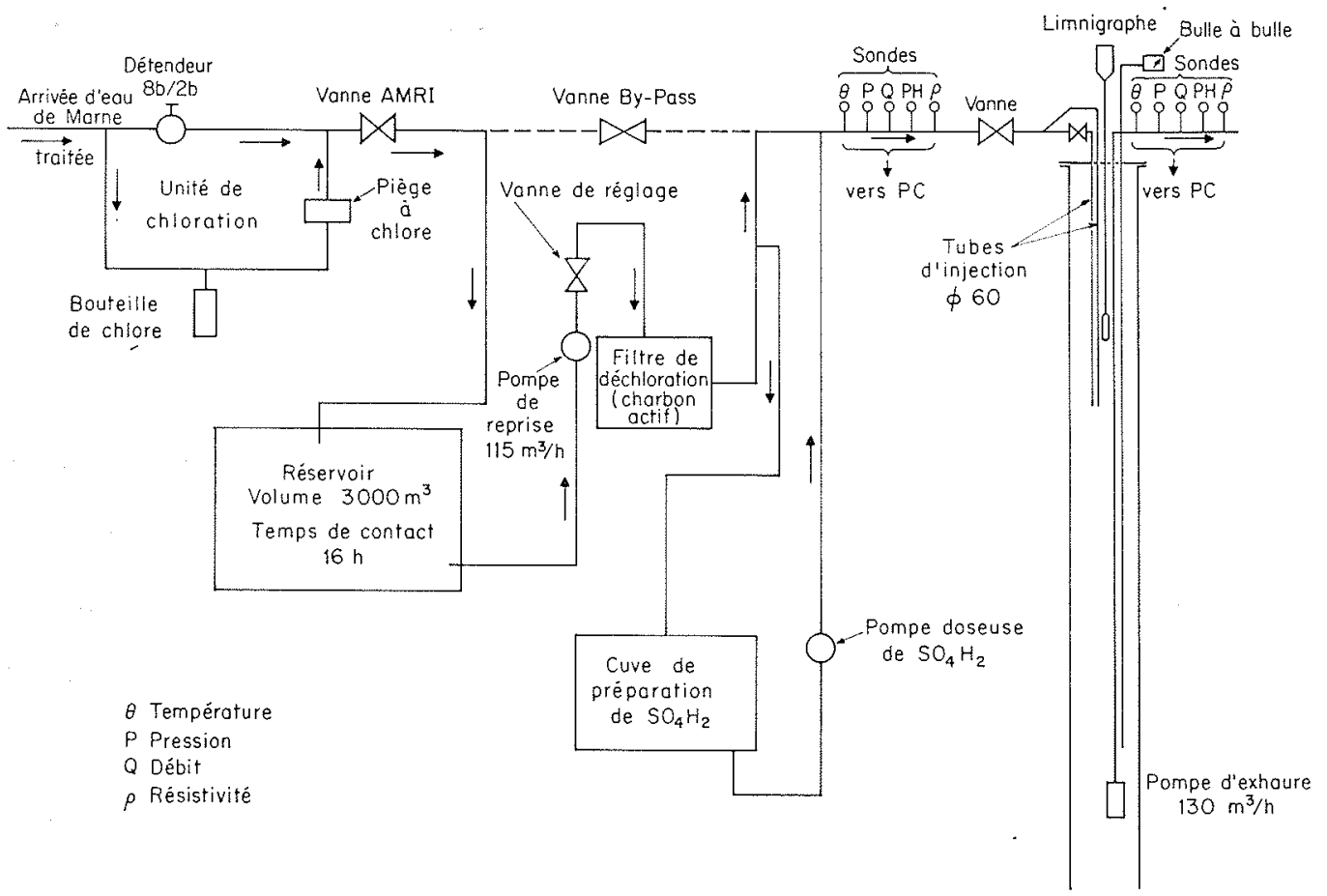

9/ Schéma de l'installation d'injection au forage de Noisy-le-Grand.

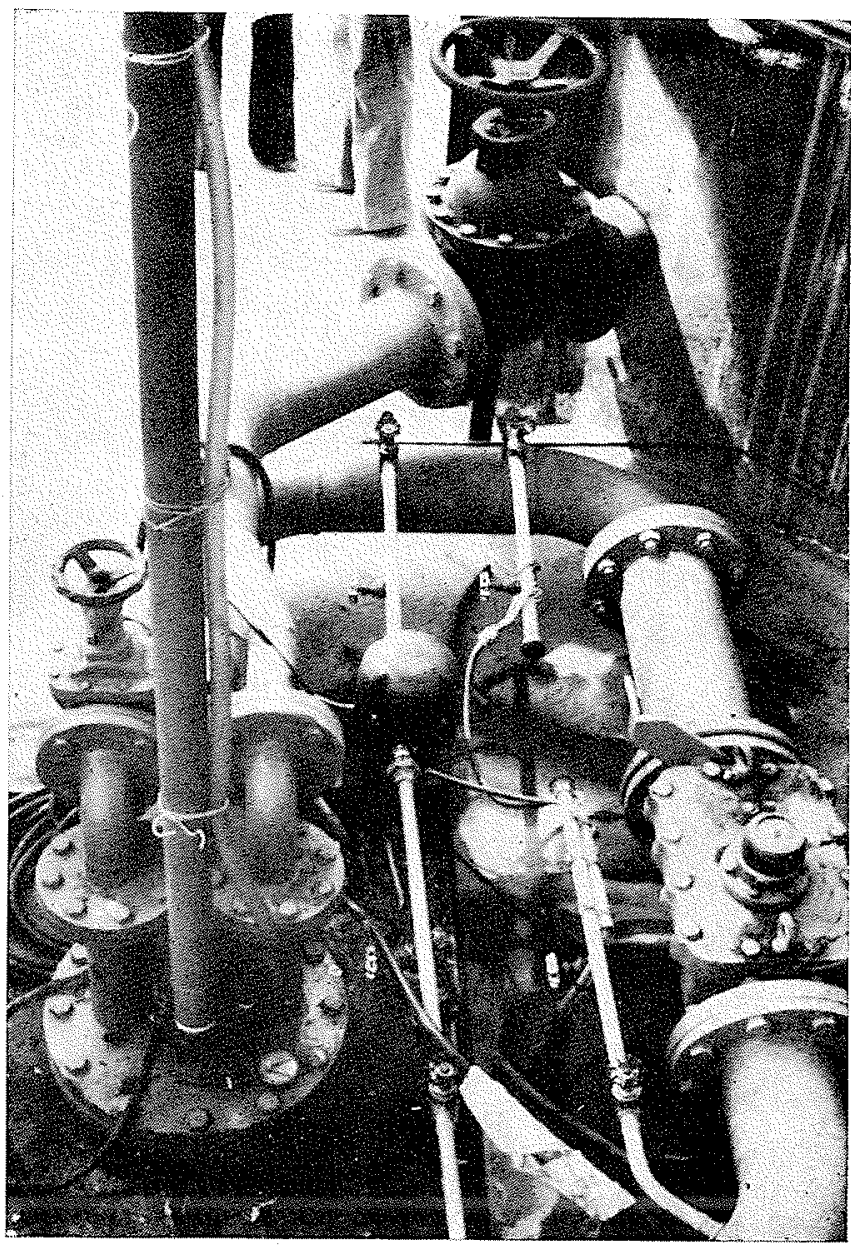

A/ Vue de la tête du forage de Noisy-le-Grand. 
parisienne (à ce titre, un questionnaire a été fourni à tous les exploitants leur demandant de nous renseigner sur le débit et le niveau de leur forage depuis l'été 1972);

- des variations de pression atmosphérique (mais leur influence est négligeable, les variations de niveau dans les forages de l'Albien se comptant, en centimètre, grossièrement comme la moitié de la variation en millibar);

- enfin, de la température des eaux injectées, qui à un double effet, d'une part sur la viscosité de ces eaux donc de la perméabilité de l'aquifère à ces mêmes eaux, d'autre part sur leur densité, donc sur la hauteur de la colonne d'eau dans le forage. Notons que les corrections de température étaient parmi les plus importantes. De $5^{\circ} \mathrm{C}$ (température de l'eau d'injection en janvier) à $32{ }^{\circ} \mathrm{C}$ (température des eaux de gisement), la dilatation de la colonne d'eau du forage s'élève à $3,5 \mathrm{~m}$. La variation de transmissivité est plus difficile à déterminer, la température moyenne apparente du mélange dans l'aquifère échappant aux mesures directes.
Précisons enfin, que pour la mise en évidence du colmatage, on possédait d'autres moyens d'approche: tests d'arrêt d'injection et tests de pompages.

Sans entrer dans le détail des différentes corrections et de la mise en œuvre de ces tests, ce qui nous ferait sortir du cadre de cet exposé, nous présenterons les résultats les plus évidents et les plus significatifs de l'essai.

\section{Résultats de l'essai (fig. 10 et 11)}

Le fait principal à noter d'emblée est qu'aucun colmatage perceptible ne s'est manifesté durant les six mois d'injection. Déjà, l'allure assez régulière et monotone de la couche de remontée brute (fig. 10) montrait qu'en tout état de cause il n'existait aucun colmatage intempestif comme il arrive assez fréquemment sur les forages d'injection. Par ailleurs, les tests ont confirmé ce résultat; ils ont montré, en outre, qu'on ne pouvait pas conclure non plus qu'il existait un colmatage progressif "vieillissant» le forage.

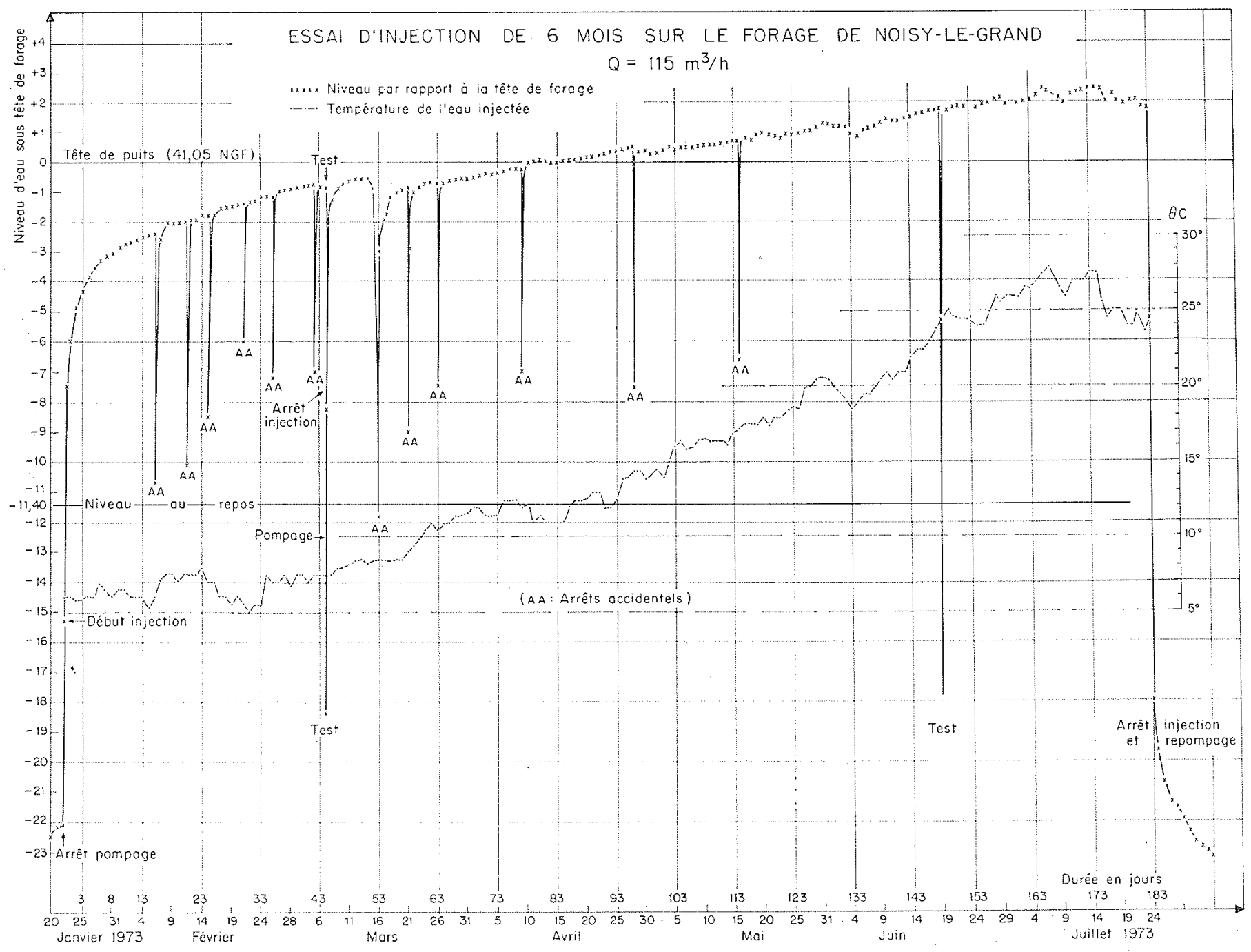

10/ Remontée de pression au forage d'injection. Température des eaux injectées. 


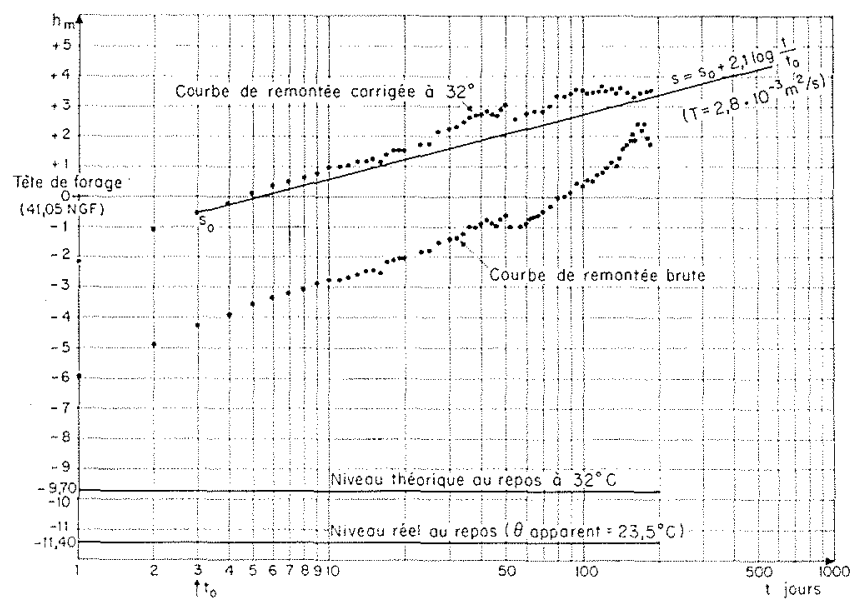

11 / Interprétation de la remontée de pression au forage.

En définitive, sans qu'il soit toutefois possible de se prononcer sur le devenir de phases d'injection supérieures à six mois, mais justement compte tenu de la durée non négligeable d'une telle période, ce résultat est très encourageant pour la mise en œuvre du projet. On note, de plus, que l'arrêt de la stérilisation par surchloration n'a eu aucun effet sur la capacité d'injection du forage. C'est donc seulement eu égard au maintien de la qualité du gisement que pourrait se justifier un tel «super-traitement».

Une nouvelle manière d'approcher les résultats de l'essai consiste à reporter les valeurs de remontée en coordonnées semi-logarithmiques. La figure 11 présente deux courbes :

- la courbe brute, sans correction de température, qui intéresse l'exploitant pour la prévision des remontées aut forage. Dans co but, il est indispensable de la lire par rapport à un axe des abcisses représentant le niveau réel de l'eau aul repos dans le forage, soit à $-11,40 \mathrm{~m}$ sous la tête de forage. (Ce niveau correspond à une répartition géothermique de la température dans la colonne d'eau du forage, soit, en définitive à une température moyenne de $22,5^{\circ} \mathrm{C}$ ou encore à une densité moyenne de 0,9975 , qui est celle de l'eau à $23,5^{\circ} \mathrm{C}$ ).

On constate que pour un débit de $115 \mathrm{~m}^{3} / \mathrm{h}$ et une température de l'eau injectée passant de $5,5^{\circ} \mathrm{C}$ à quelque $26^{\circ} \mathrm{C}$ en fin d'essai, le relèvement au bout de siy mois est de $14 \mathrm{~m}$ dans le forage.

- la courbe corrigée des effets de densité et rapportée à $32^{\circ} \mathrm{C}$ (température de l'eau du gisement), qui se lit par rapport à un niveau au repos à $32^{\circ} \mathrm{C}$ soit $-9,70 \mathrm{~m}$ sous la tête de forage.

Nous avons tracé, par ailleurs, la droite tirée de la formule de Jacob:

$s=[0,183 Q / T]\left[\log \left(2,25 T / / r^{2} S\right)\right]+J \quad$ (pertes de charge)

où les paramètres hydrauliques adoptés sont ceux du pompage donc à une température de $32^{\circ} \mathrm{C}$ et qui représente les relèvements théoriques au forage. La différence entre les deux courbes donne l'ordre de grandeur de la perte de charge due à la diminution de transmissivité provoquée par l'injection d'eau froide. Cette perte ne semble pas dépasser $1 \mathrm{~m}$.

Si maintenant on compare la courbe brute et la courbe à $32{ }^{\circ} \mathrm{C}$, on constate que cette perte de charge est beaucoup plus faible que le gain de charge dû aux effets de densité provoqués par la faible température des eaux injectées.

Or, la profondeur du niveau piézométrique de la nappe albienne sous la région parisienne n'est pas très importante, ce qui peut conduire à la nécessité d'injecter en mettant en cuvre des dispositifs spéciaux, si le niveau dynamique s'établit au-dessus du sol. L'essai de Noisy montre qu'injecter hors saison chaude - donc en période de faible demande, lorsqu'il existe a priori des eaux potables excédentaires - constitue une donnée favorable du projet.

\section{Discussion}

M. le Président remercie M. Brze pour son exposé, qui complète celui de M. Plaud, et ouvre la discussion.

M. Durousseau souhaite quelques précisions sur les deux points suivants :

$1^{\circ}$ Pourquoi envisage-t-on l'utilisation de doublets on de quadriplets pour l'injection et l'extraction? Les forages existants ne sont-ils pas suffisants? A-t-on des doutes sur la capacité de transmission des pressions de la nappe?

$2^{\circ}$ Vos essais montrent-ils, pour un même forage, des caractéristiques (débit spécifique, notamment) identiques en régime d'injection et en régime d'extraction?

Sur le premier point, M. Bize observe que la nappe de l'Albien constitue :

a) une nappe intéressante pour des exploitants de plus en plus nombreux;

b) une nappe "gelée », en ce sens que le Service des Mines, appliquant le décret-loi de 1935, interdit tout nouveaul forage susceptible d'abaisser le niveau piézométrique; c) une ressource qui se prêterait bien à une exploitation progressive à mesure du développement des villes nouvelles en cours de création dans la région parisienne; il est possible qu'une telle exploitation soit plus rentable que l'établissement d'une grosse conduite d'adduction deau superficialle - par exemple grevé, dans une première phase d'exploitation, par le poids d'intérêts intercalaires importants.

Si, par un système de doublets, il est possib'e d'ouvrir un nouveau forage dans l'Albien, à condition de compenser son action sur la nappe par un forage d'injection placé à quelque distance auprès d'une station de traitement ou d'une grosse artère de distribution, il est vraisembable que l'on aboutira à une so!ution particulièrement économique.

En ce qui concerne la deuxième question posée par $M$. DurousSEAU, je dirai, quavant nos essais, nous croyions - sur la base d'expériences faites en milieu sableux - que le débit spécifique «en injection » était plus faible que le débit spécifique «en pompage »; cela a été assez souvent constaté, notamment sur les nombreux forages dans la nappe de la plaine côtière en Israël. Dans notre cas, 
compte tenu du fait qu'à Noisy il existe un «développement» assez considérable autoux du forage, nous avons pu mettre en évidence qu'il y avait une réversibilité à peu près parfaite des débits spécifiques d'injection et de pompage.

M. Degallyer revient sur la question de la «drainance»

M. Plaud, dit-il, nous a montré une carte de la nappe de Bordeaux en 1880; déjà à cette époque, on pouvait constater une forte action de la topographie des grands fleuves sur la topographie du nivean piézométrique de la nappe profonde. Pour la nappe de l'Albien, je me rappelle avoir vu des documents oì l'on voyait exactement le même phénomène : avant toute exploitation, la vallée de la Seine et celle de la Somme, avaient déjà, sur le niveau de la nappe de l'Albien, une influence de 30 à $40 \mathrm{~m}$ de rabattement; autrement dit, il y avait, dès cette époque, un très fort débit naturel vers le haut de l'Albien, celui-ci alimentant dans les va'lées, la nappe de la craie. Aujourdhui, la nappe de l'Albien a vu son niveau piézométrique abaissé d'une centaine de mètres dans la région de Paris, région où, précisément, on avait constaté jadis un rabattement initial important; autrement dit, le sens de l'écoulement dans la zone de drainance a été inversé. Mais vous avez dit que cet effet était tenu pour négligeable.

Cela me semble assez grave, parce que, avec le système des doublets, on va pomper dans une zone peu exploitée et injecter dans une zone đéjà exploitée. On va étenđre l'entonnoir piézométrique de la nappe de l'Albien, donc faire un appel supplémentaire à la nappe de la craic.

Ces appels à la nappe de la craie vont se répercuter jusqu'à la surface piézométrique de celle-ci et on va assister au tarissement de nombreuses rivières dans la craie. La craje s'alimente souvent assez profondément au-dessous de la surface du sol et je ne pense pas qu'un abaissement du niveau de la nappe de la craie entraînera une augmentation de son alimentation.

Le cas est peut-être différent dans la région de Bordeaux où, dans certains secteurs, les nappes ont un trop-plein qu'on pourra réduire en vue d'obtenir ainsi un supplément d'alimentation. Dans la région de Paris, un tel supplément n'existe pas; on se trouvera ainsi amené à utiliser un jour le système préconisé par les AngloSaxons qui, n'ayant pas la chance d'avoir de grandes rivières, envisagent, dès maintenant, non plus de rechercher des nappes souterraines, mais de les surexploiter systómatiquement afin de les déconnecter des rivières qu'elles alimentent jusqu'à présent.

Je pense qu'on ne se ralliera pas tout de suite à ce procédé en France; mais, on peut prévoir que, dans un avenir de cinc à dix ans, on va voir se généraliser des instatlations de recharge artificielle de nappes; au-delà de cette période, ce sont des alimentations de rivières qui seront faites aux dépens des nappes, en vue de maintenir un minimum de débit dans lesdites rivières: à ce moment-là, l'alimentation artificielle n'aura plus de raison d'être puisqu'on n'aura pius d'eau de surface disponible.

La question est très complexe, répond $M$. BIzL, mais je n'ai encore jamais vu un hydrogéologue arguer de la drainance pour modifier l'exploitation d'une nappe captive, Le problème se pose partout. Je crois que nous devrons tous parler de la drainance le jour où lon aura vu une nappe captive profonde intensément surexploitée pour savoir exactement ce qui se passe.

Qu'avons-nous à notre disposition pour étudier la drainance? Une courbure, une discontinuité dans une courbe de "remontée » ou de «pompage» et bien des mécanismes peuvent être invoqués pour interpréter cette observation. Il est certain qu'il y a des phénomènes de drainance, mais, quant à moi, je me refuse pour le moment à arguer de la drainance pour prendre des options d'exploitation industrielle sur une nappe captive, et je crois que je ne suis pas le seul.

Ceci dit, dans le cas de l'Albien, il y a, peut-être en raison des innombrables obscurités sur l'alimentation de la nappe, un souci de conserver une moyenne - sans variations d'amp'itudes importantes au niveau de Paris. Donc, je ne pense pas que, du point de vue de l'ensemble des ressources de la région parisienne, le projet puisse modifier quoi que ce soit de l'ensemble de ces ressources. C'est bien là l'objet de l'étude.

II a été démontré, par le Service des Mines, qu'exploiter à outrance la nappe de l'Albien, et rabattre même son niveau de plus de $80 \mathrm{~m}$ est économiquement rentable. On part d'un taux d'actualisation de $10 \%$ c'est une affaire bien plus rentable que l'opération d'alimentation artificielle. Le souci de la conservation de la ressource, et d'une ressource de bonne qualité, justifie la position actuelle qui est une position de statu quo, mais avec un recours a lalimentation artificielle permettant d'utiliser cette nappe de l'Albien. Le niveau de celle-ci n'ayant pas varié en moyenne, il n'y aura rien do changé.

M. le Président se déclare d'accord dans les grandes lignes avec les conceptions de M. BIze, mais, observe-t-il, dans la politique d'exploitation d'une nappe captive, la conservation des niveaux peut être un objectif, mais, il y en a d'autres; actue!lement, l'objectif est la conservation des niveaux; ce n'est pas la conservation de la ressource, ni de l'optimisation de son utilisation.

Dans votre projet, on envisage de se servir d'un système de doublets de forage en nappe captive, en comptant utiliser l'aquifère à la fois comme vecteur et comme modulateur. Un aquifere peut, difïicilement, être à la fois un bon vecteur et un bon conduc teur : il est soit l'un, soit l'autre. Dans le cas présent, il s'agit d'une nappe captive dans laquelle il n'est pas possible d'accumuler de gros volumes d'eau : il s'agira de «stockage de pression» plus que de stockage d'eau, et e'est surtout la fonction conductrice de l'aquifère qu'on va utiliser et faire fonctionner en régime quasi permanent.

En effet, dit M. BIzE, c'est le rô'e de «vecteur'conduite » qui prime et on s'aperçoit, chemin faisant, qu'en plus on a la possibilité de stocker à un certain moment de l'eau potab'e.

M. de Marsily (Ecole des Mines, Fontainebleau) intervient en ces termes :

Les calculs que vous avez présentés sont, dites-yous, faits à la main, mais vous envisagez d'utiliser un modèle.

Pourriez-vous nous dire quels résultats vous attendez de ce modèle?

Ne pensez-vous pas, d'autre part, que la construction d'un modèlo multicouche, tel que celui bâti en Aquitaine par l'Ecole des Mines, dont à parlé M. PLAUD, permettrait de lever partiellement les incertitudes sur la drainance évoquée par M. DEGAllier?

Sur le premier point, M. BIzE apporte la réponse ci-après :

Le premier objectif de notre modèle est de montrer que la politique du Service des Mines, qui était antérieurement une politique de «gel» et de statu quo, peut devenir une politique de souplesse et amener une nouvelle formulation de textes rigoureux; le modèle sera le moyen de décider; dans tel cas cela peut marcher, dans tel autre, il vaut mieux s'abstenir. C'est à partir de ce processus pratique et souple que des autorisations pourront être données.

Le second objectif est de montrer ce qui va se passer, à la suite, par exemple, de l'implantation d'un doublet de prélèvement et d'injection à côté d'une exploitation existante. Il y a une solidarité qui va se faire jour dans la nappe qui existe, bien sût, déjà, mais dans laquelle on va modifier son exploitation et hon son niveau; le modèle permettra de montrer aux divers exploitants ce qui peut se présenter; des calculs seront faits pour savoir si l'on a intérêt, économiquement, à commencer à pomper d'abord et à injecter le plus tard possible pour boucher la dépression crée initialement, ou retrouver un certain niveau de pression à un endroil donné; on étudiera les transitoires et on verra jusqu'où on peut aller avec des hypothèses diverses d'injection ou de pompage. C'est un outil très souple qui peut donner des résultats d'ici quelques mois.

A propos de la seconde partie de l'intervention de M. de MaRSILY, M. BIZE précise.

Nous n'envisageons pas de faire le modèle de l'Albien. Notre modèle est un modèle d'exploitation à objectif très limité; il donnera des ordres de grandeur; mais, délibérément, nous n'envisageons pas de représenter l'Albien dans sa totalité et, le cas échéant, de résoudre le problème que vous évoquez; cela pourra être envisagé plus tard.

M. le Président clôt la discussion en rappelant que, dans les problèmes tels que ceux traités par M. BızE, il ne faut pas omettre de faire intervenir la température de l'eau.

11 donne ensuite la parole à M. Busse pour l'exposé de sa communication. 